\title{
Porous Se@SiO nanocomposites protect the femoral head from methylprednisolone-induced osteonecrosis
}

This article was published in the following Dove Press journal:

International Journal of Nanomedicine

\section{Guoying Deng',*, Chenyun $\mathrm{Dai}^{2}, *$, Jinyuan Chen', Anqi ji', Jingpeng Zhao', Yue Zhai', Yingjie Kang ${ }^{3}$, Xijian Liu $^{4}$, Yin Wang ${ }^{5}$, Qiugen Wang'}

'Trauma Center, Shanghai General Hospital, Shanghai Jiaotong University School of Medicine, Shanghai, China; 'Institute of Translation Medicine, Shanghai General Hospital, Shanghai Jiaotong University School of Medicine, Shanghai, China; ${ }^{3}$ Department of Radiology, Shuguang Hospital, Shanghai University of Traditional Chinese Medicine, Shanghai, China; ${ }^{4}$ College of Chemistry and Chemical Engineering, Shanghai University of Engineering Science, Shanghai, China; ${ }^{5}$ Ultrasound Department of Shanghai Pulmonary Hospital, Tongji University, Shanghai, China

*These authors contributed equally to this work
Correspondence: Qiugen Wang Trauma Center, Shanghai General Hospital, Shanghai Jiaotong University School of Medicine, 650 Xin Songjiang Road, Shanghai 201620, China

Tel +86 | 33। 1986527

Email wangqiugen@।26.com

Yin Wang

Ultrasound Department of Shanghai Pulmonary Hospital, Tongji University, No. 507, Zhengmin Road, Yangpu District, Shanghai, 200433, China

Tel +86 I 370 I88 5। 38

Email Ipbbl@aliyun.com
Background: Methylprednisolone (MPS) is an important drug used in therapy of many diseases. However, osteonecrosis of the femoral head is a serious damage in the MPS treatment. Thus, it is imperative to develop new drugs to prevent the serious side effect of MPS.

Methods: The potential interferences $\mathrm{Se} @ \mathrm{SiO}_{2}$ nanocomposites may have to the therapeutic effect of methylprednisolone (MPS) were evaluated by classical therapeutic effect index of acute respiratory distress syndrome (ARDS), such as wet-to-dry weight ratio, inflammatory factors IL- $1 \beta$ and TNF- $\alpha$. And oxidative stress species (ROS) index like superoxide dismutase (SOD) and glutathione (GSH) were tested. Then, the protection effects of $\mathrm{Se} @ \mathrm{SiO}_{2}$ have in osteonecrosis of the femoral head (ONFH) were evaluated by micro CT, histologic analysis and Western-blot analysis.

Results: In the present study, we found that in the rat model of ARDS, $\mathrm{Se}\left(\mathrm{SiO}_{2}\right.$ nanocomposites induced SOD and GSH indirectly to reduce ROS damage. The wet-to-dry weight ratio of lung was significantly decreased after MPS treatment compared with the control group, whereas the $\mathrm{Se} @ \mathrm{SiO}_{2}$ did not affect the reduced wet-to-dry weight ratio of MPS. Se@ $\mathrm{SiO}_{2}$ also did not impair the effect of MPS on the reduction of inflammatory factors IL- $1 \beta$ and TNF- $\alpha$, and on the alleviation of structural destruction. Furthermore, micro CT and histologic analysis confirmed that $\mathrm{Se} @ \mathrm{SiO}_{2}$ significantly alleviate MPS-induced destruction of femoral head. Moreover, compared with MPS group, $\mathrm{Se} @ \mathrm{SiO}_{2}$ could increase collagen II and aggrecan, and reduce the IL-1 $\beta$ level in the cartilage of femoral head. In addition, the biosafety of $\mathrm{Se} @ \mathrm{SiO}_{2}$ in vitro and in vivo were supported by cell proliferation assay and histologic analysis of main organs from rat models.

Conclusion: $\mathrm{Se} @ \mathrm{SiO}_{2}$ nanocomposites have a protective effect in MPS-induced ONFH without influence on the therapeutic activity of MPS, suggesting the potential as effective drugs to avoid ONFH in MPS therapy.

Keywords: porous $\mathrm{Se} @ \mathrm{SiO}_{2}$ nanocomposites, methylprednisolone, osteonecrosis of femoral head, ROS damage, ARDS

\section{Introduction}

Methylprednisolone (MPS) plays an irreplaceable role in the treatment of various diseases, including rheumatoid arthritis, systemic lupus erythematous, and acute respiratory distress syndrome (ARDS). However, MPS-induced osteonecrosis of the femoral head (ONFH) is an irreversible and devastating injury. ${ }^{1}$ The only treatment option is hip replacement. ${ }^{2}$ Thus, there is an urgent need to reduce the risk of ONFH during MPS treatment.

Many studies focus on the risk factors for MPS-induced ONFH. Among them, oxidative stress disorders are a vital concern. ${ }^{3,4}$ Many antioxidants have proven to be effective 
in therapy for steroid-induced osteonecrosis in rat models., ${ }^{5,6}$ It is reported that selenium (Se), an essential micronutrient in humans and animals with a wide range of biologic functions, ${ }^{7}$ could suppress oxidative stress and serves as a reactive oxygen species (ROS) scavenger. ${ }^{8-11}$ In some cases, a deficiency of Se could induce oxidative stress and endoplasmic reticulum stress. ${ }^{12,13}$ Due to a narrow margin between beneficial and toxic effects, Se is not safe to use in clinical treatment. ${ }^{14,15} \mathrm{By}$ using nanotechnology, Se particles are modified to develop $\mathrm{Se} @ \mathrm{SiO}_{2}$ nanocomposites. Accumulating evidence shows that $\mathrm{Se} @ \mathrm{SiO}_{2}$ nanocomposites act as antioxidants with reduced toxicity. ${ }^{16,17}$ Thus, we speculated that $\mathrm{Se} @ \mathrm{SiO}_{2}$ nanocomposites might serve as a cytoprotectant in MPS-induced ONFH due to their ability to reduce ROS production.

In this study, we investigated whether $\mathrm{Se} @ \mathrm{SiO}_{2}$ influenced the therapeutic ability of MPS as well as the femoral head necrosis induced by MPS in rat models. The possible mechanisms underlying the effect of $\mathrm{Se} @ \mathrm{SiO}_{2}$ on MPSinduced femoral head necrosis were also explored.

\section{Materials and methods}

\section{Synthesis and characterization of porous $\mathrm{Se} @ \mathrm{SiO}_{2}$ nanocomposites}

The detailed synthesis procedures of porous $\mathrm{Se} @ \mathrm{SiO}_{2}$ nanocomposites were performed according to the methods reported in our previous study. ${ }^{18}$ Briefly, $\mathrm{Cu}_{2-\mathrm{x}}$ Se nanocrystals were first prepared and mixed with $n$-hexane, $n$-hexanol, Triton X-100, deionized water, and tetraethyl orthosilicate. $\left[\mathrm{Cu}\left(\mathrm{NH}_{3}\right)_{4}\right]^{2+}$ was developed by the addition of ammonium hydroxide to the mixture. Oxygen was used to oxidize $\mathrm{Se}^{2-}$ to develop Se quantum dots. The silica coated the Se quantum dots by orthosilicate hydrolysis in an alkaline environment, forming solid $\mathrm{Se} @ \mathrm{SiO}_{2}$ nanocomposites. Then, the solid $\mathrm{Se} @ \mathrm{SiO}_{2}$ nanocomposites were coated with PVP and etched in hot water to construct porous structures. Se@ $\mathrm{SiO}_{2}$ nanocomposites were characterized by means of a D/max-2550 PC X-ray diffractometer $(\mathrm{XRD}, \mathrm{Cu}-\mathrm{K} \alpha$ radiation; Rigaku; Tokyo, Japan) and transmission electronic microscopy (JEM-2100F). The cumulative release kinetics of Se from the porous Se@SiO, nanocomposites in $\mathrm{PBS}$ at $37^{\circ} \mathrm{C}$ with different $\mathrm{pH}$ values ( $\mathrm{pH} 7.4$ and $\mathrm{pH}$ 5.0) were consistent with those reported in our previous study. ${ }^{18}$

\section{Cell lines and animals}

The cell line RLE-6TN (rat lung epithelial-T-antigen negative) was purchased from American Type Culture Collection. Cartilage cells were derived from Sprague Dawley (SD) rats. The primary culture of the cartilage cells was performed according to the methods described in a previous study. ${ }^{19}$ Briefly, adult SD rats were sacrificed and the head of the femur was exposed under aseptic conditions. The total articular cartilage was isolated and cut into pieces (a volume of $\sim 1 \mathrm{~mm}^{3}$ ). After digestion with $0.2 \%$ collagenase type II and filtration with a cell strainer, the cartilage cells were collected and seeded on tissue culture dishes. All the cells were authenticated twice by morphologic and isoenzyme analyses during the study period. Cell lines were routinely checked for contamination by mycoplasma using Hoechst staining and consistently found to be negative. The cells were cultured with DMEM/F-12 with $10 \%$ fetal bovine serum in a $37^{\circ} \mathrm{C}, 5 \%$ $\mathrm{CO}_{2}$ environment. The medium was changed every $2-3$ days. The cartilage cells were identified by immunofluorescence staining with collagenase type II and aggrecan (all from Santa Cruz Biotechnology Inc., Dallas, TX, USA).

Eight-week-old female SD rats were purchased from the Experimental Animal Center of Shanghai Jiao Tong University. The rats were bred and maintained under a 12/12-hour light-dark cycle with free access to food and water. The temperature was maintained at $18^{\circ} \mathrm{C}-25^{\circ} \mathrm{C}$, and the relative humidity was set to $40 \%-60 \%$. Rats were housed for a week before the experiments began.

\section{In vitro safety of $\mathrm{Se} @ \mathrm{SiO}_{2}$ nanocomposites} The in vitro safety of $\mathrm{Se} @ \mathrm{SiO}_{2}$ nanocomposites was determined by the inhibitory effect of $\mathrm{Se} @ \mathrm{SiO}_{2}$ nanocomposites on cell proliferation. RLE-6TN cells and cartilage cells were seeded at a density of $6 \times 10^{3}$ cells per well in a flat-bottomed 96-well plate in a humidified $37^{\circ} \mathrm{C}$ and $5 \% \mathrm{CO}_{2}$ atmosphere. The next day, the cells were incubated with an increasing concentration of $\mathrm{Se} @ \mathrm{SiO}_{2}$ (ranging from 0 to $180 \mu \mathrm{g} / \mathrm{mL}$ ). The medium was replaced every 2 or 3 days. Five days later, the cell proliferation was determined by a Cell Counting Kit-8/WST-8 (Dojindo; Kumamoto, Japan).

\section{A rat model of ARDS}

The SD rats $(n=24)$ were randomly divided into four groups: control group, Se@ $\mathrm{SiO}_{2}$ group, MPS group, and MPS+ $\mathrm{Se} @ \mathrm{SiO}_{2}$ group. All the rats were treated with $4 \mathrm{mg} / \mathrm{kg}$ lipopolysaccharide (LPS). Half an hour later, the MPS group was intramuscularly administered $30 \mathrm{mg} / \mathrm{kg}$ MPS and the $\mathrm{Se} @ \mathrm{SiO}_{2}$ group was intraperitoneally injected with $1 \mathrm{mg} / \mathrm{kg}$ $\mathrm{Se} @ \mathrm{SiO}_{2}$. The MPS+Se@ $\mathrm{SiO}_{2}$ group received an intramuscular injection of $30 \mathrm{mg} / \mathrm{kg}$ MPS and an intraperitoneal injection of $1 \mathrm{mg} / \mathrm{kg} \mathrm{Se} @ \mathrm{SiO}_{2}$. Six hours later, the rats were sacrificed by an intraperitoneal administration of $1 \%$ sodium pentobarbital ( $40 \mathrm{mg} / \mathrm{kg})$. The anterior lobe and the middle lobe of the right lung were excised to determine the wet-to-dry weight ratio. The other lobes of the right lung were cryopreserved 
in liquid nitrogen for further study. Tissues from the left lung lobe, femoral head, heart, liver, spleen, and kidney were kept in neutral formalin solution and embedded in paraffin for morphologic examination. The animal experiments were approved by the Institutional Animal Care and Use Committee of Shanghai Jiao Tong University. All the animals were treated in accordance with the guidelines of the Institutional Animal Care and Use Committee of Shanghai Jiao Tong University.

\section{A rat model of femoral head osteonecrosis}

Male SD rats ( $\mathrm{n}=18 ; 6$ rats in each group) were randomly divided into a blank group (blank), a vehicle group (vehicle), and a porous $\mathrm{Se} @ \mathrm{SiO}_{2}$ nanocomposite group (NP). The SD rats from the vehicle and NP groups were intravenously injected with $10 \mu \mathrm{g} / \mathrm{kg}$ LPS. After $24 \mathrm{~h}$, these SD rats received three injections of MPS $(20 \mathrm{mg} / \mathrm{kg})$ intramuscularly every day. The NP group was also injected intraperitoneally with porous $\mathrm{Se} @ \mathrm{SiO}_{2}$ nanocomposites $(1 \mathrm{mg} / \mathrm{kg}$ ) per day. The blank group received saline injections and was housed under identical conditions. To prevent infection, each rat was administered 100,000 U of penicillin intramuscularly. The rats in all the groups were sacrificed 8 weeks after the first MPS injection. The femoral heads of SD rats were harvested. The left femoral heads were preserved in a $-70^{\circ} \mathrm{C}$ cryogenic freezer immediately after sacrifice, and the proteins were isolated for Western blot analysis. The right femoral heads were collected and immediately fixed with $10 \%$ formalin (0.1 M phosphate buffer, $\mathrm{pH} \mathrm{7.4)}$ at $4{ }^{\circ} \mathrm{C}$ for $24 \mathrm{~h}$. Then, the samples were used for a micro-computed tomography (Micro-CT) scan and histologic analysis following previous protocols. ${ }^{20}$

\section{Glutathione (GSH) and superoxide dismutase (SOD) in lung tissues}

Rat lung tissues or cartilages from different groups were lysed in RIPA lysis buffer (Beyotime, Shanghai, China). After centrifuge for $15 \mathrm{~min}$ in the speed of $2500 \mathrm{rpm}$, the levels of glutathione (GSH) and superoxide dismutase (SOD) were detected with reduced glutathione assay kit (Spectrophotometric method) and superoxide dismutase assay kit (WST-1 method), respectively following the instructions provided by manufacturer.

\section{Wet-to-dry weight ratio of the lung tissue}

The anterior lobe and the middle lobe of the right lung were excised. The immediate weight was the wet weight. Then, the lungs were heated at $70^{\circ} \mathrm{C}$ for 2 days. Once the weight was found to be constant, it was measured and recorded as the dry weight. The wet-to-dry weight ratio was calculated by dividing the wet weight by the dry weight.

\section{Western blot}

Rat lung tissues or cartilages from different groups were preserved in $\mathrm{a}-70^{\circ} \mathrm{C}$ cryogenic freezer immediately after sacrifice, and the proteins were isolated for Western blot analysis. The concentrations of proteins were detected by a BCA protein assay kit (Beyotime). The protein samples were subjected to sodium dodecyl sulfate-polyacrylamide gel electrophoresis. Then, the proteins were transferred to polyvinylidene difluoride membranes and immunoblotted with the indicated primary antibody. GAPDH served as a loading control. The primary antibodies were all purchased from Cell Signaling Technology (Boston, MA, USA). After washing three times with TBST, the membranes were incubated with a horseradish peroxidase-conjugated secondary antibody (all from Cell Signaling Technology) for $1 \mathrm{~h}$ at room temperature. The proteins were visualized using enhanced chemiluminescence reagents (Plus-ECL; PerkinElmer Inc., Waltham, MA, USA).

\section{Histologic analysis}

Tissues of rats from different groups were fixed in a neutral formalin solution and were processed for paraffin sectioning. Sections of $\sim 5 \mu \mathrm{m}$ thickness were stained with H\&E or collagen type II. The sections were observed under a light microscope (Leica DM5500 B; Leica Microsystems, Wetzlar, Germany).

\section{Micro-CT procedure}

A Micro-CT (GE Healthcare Biosciences, Piscataway, NJ, USA) was used to detect changes in the excised femoral head samples.

\section{Statistical analysis}

All the values are presented as the mean \pm standard error of mean $(n=6)$. Significant differences between groups were determined with GraphPad Prism 5 (GraphPad Software Inc., San Diego, CA, USA) using Student's unpaired $t$-test. Differences were considered significant at $P<0.05$.

\section{Results}

\section{Characterization of porous $\mathrm{Se} @ \mathrm{SiO}_{2}$ nanocomposites}

We used the XRD pattern to assess the phase structure of the solid $\mathrm{Se} @ \mathrm{SiO}_{2}$ nanocomposites. Many well-defined characteristic peaks in solid $\mathrm{Se} @ \mathrm{SiO}_{2}$ nanocomposites showed the hexagonal phase, referenced by the standard Se phase (JCPDS card no 65-1876; Figure 1A). The XRD pattern of the solid $\mathrm{Se} @ \mathrm{SiO}_{2}$ nanocomposites exhibited an increase in 

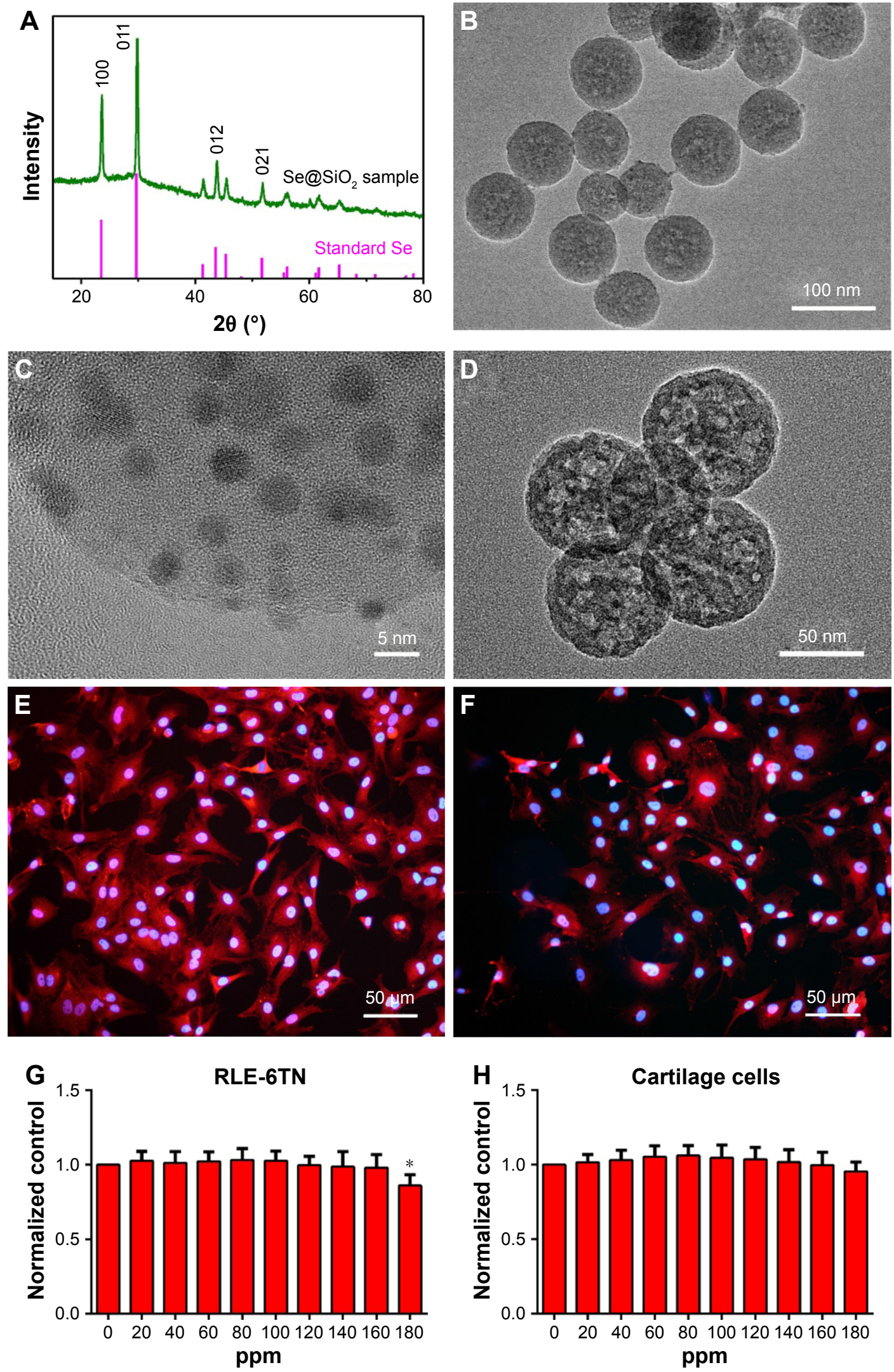

Figure I Characterization of $\mathrm{Se} @ \mathrm{SiO}_{2}$ nanocomposites.

Notes: (A) XRD pattern of the solid Se@SiO, nanocomposites and the standard hexagonal phase of Se (JCPDS card no: 65- I876). (B) Low and (C) high magnification TEM

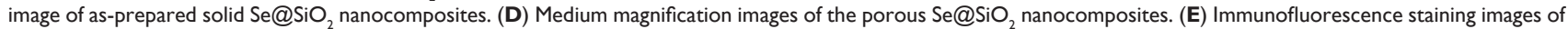
cartilage cells with DAPI and collagenase type II (F). Immunofluorescence staining images of cartilage cells with DAPI and aggrecan. (G, H) The cell viability of RLE-6TN cells (G) and cartilage cells $(\mathbf{H})$ incubated with different concentrations of $\mathrm{Se} @ \mathrm{SiO}_{2}$ nanocomposites for 24 h. $* \mathrm{P}<0.05$ compare to control $(0$ ppm).

Abbreviations: TEM, transmission electronic microscopy; XRD, X-ray diffractometer. 
the low angle region, which may be attributable to amorphous silica (Figure 1A).

The morphology and size of the $\mathrm{Se} @ \mathrm{SiO}_{2}$ nanocomposites were determined by transmission electronic microscope. Figure $1 \mathrm{~B}$ and $\mathrm{C}$ shows solid $\mathrm{Se} @ \mathrm{SiO}_{2}$ nanocomposites and Figure 1D shows porous $\mathrm{Se} @ \mathrm{SiO}_{2}$ nanocomposites. Their diameter was $\sim 55 \mathrm{~nm}$, and there were many small quantum dots interspersed from the center to the surface (Figure 1B). The small quantum dots were irregular, and their size was $<5 \mathrm{~nm}$ (Figure $1 \mathrm{~B}$ and C). In addition, the in vitro stability of $\mathrm{Se} @ \mathrm{SiO}_{2}$ nanocomposites and the slow release of Se quantum dots from porous $\mathrm{Se} @ \mathrm{SiO}_{2}$ nanocomposites were confirmed in our previous study.

The Cell Counting Kit-8 assay was used to determine the cytotoxicity of the $\mathrm{Se} @ \mathrm{SiO}_{2}$ nanocomposites on RLE-6TN cells and cartilage cells. The cartilage cells were identified by immunofluorescence staining with collagenase type II and aggrecan (Figure 1E and F). The cells were incubated with an increasing concentration of $\mathrm{Se} @ \mathrm{SiO}_{2}$ nanocomposites, ranging from 0 to $180 \mu \mathrm{g} / \mathrm{mL}$ (Figure $1 \mathrm{G}$ and $\mathrm{H}$ ). Compared with the negative control group, the cell viabilities of RLE6TN cells treated with $\mathrm{Se} @ \mathrm{SiO}_{2}$ nanocomposites did not decrease significantly until the concentration of $\mathrm{Se} @ \mathrm{SiO}_{2}$ nanocomposites reached $180 \mu \mathrm{g} / \mathrm{mL}$ (Figure $1 \mathrm{G}$ ). In the cartilage cells, no significant difference was observed in the cell viabilities among all the groups with $\mathrm{Se} @ \mathrm{SiO}_{2}$ nanocomposite treatment (Figure 1H).

\section{Porous Se@SiO, nanocomposites do not impair the therapeutic efficacy of MPS in a rat model of ARDS}

As shown in Figure 2, both $\mathrm{Se} @ \mathrm{SiO}_{2}$ and MPS induced SOD and GSH-PX, compared with the control group. The levels of SOD and GSH-PX in the MPS plus $\mathrm{Se} @ \mathrm{SiO}_{2}$ group were similar to those in the MPS group (Figure 2A and B). The wet-to-dry weight ratio was significantly decreased in the MPS group compared with the $\mathrm{Se} @ \mathrm{SiO}_{2}$ group, whereas the wet-to-dry weight ratio in the MPS plus Se@ $\mathrm{SiO}_{2}$ group was comparable to that in the MPS group (Figure 2C). Western blot was also used to determine the inflammation factors including interleukin (IL)- $1 \beta$ and tumor necrosis factor-alpha (TNF- $\alpha$ ) in the control group, Se $@$ $\mathrm{SiO}_{2}$ group, MPS group, and MPS+Se@ $\mathrm{SiO}_{2}$ group. The results showed that compared with the control group, Se@ $\mathrm{SiO}_{2}$ did not significantly decrease the levels of IL-1 $\beta$ and TNF- $\alpha$ (Figure 2D-F). However, MPS dramatically reduced the IL- $1 \beta$ level and TNF- $\alpha$ level (Figure 2D-F). The addition of $\mathrm{Se} @ \mathrm{SiO}_{2}$ to MPS did not increase or decrease the levels of IL- $1 \beta$ and TNF- $\alpha$ in the MPS group (Figure 2D-F). Thus, $\mathrm{Se} @ \mathrm{SiO}_{2}$ nanocomposites did not influence the therapeutic efficacy of MPS.

We also used histologic analysis to evaluate the damage in the structure of the lung tissues in the rat model of ARDS. We observed alveolar hemorrhage, edema, and inflammatory cell infiltration (Figure 3). MPS markedly alleviated the lung damage, whereas $\mathrm{Se} @ \mathrm{SiO}_{2}$ did not alter the effect of MPS on the injured lung tissues (Figure 3).

\section{$\mathrm{Se} @ \mathrm{SiO}_{2}$ nanocomposites reduce MPS- induced osteonecrosis in femoral head}

A Micro-CT scan was used to determine the therapeutic effect of $\mathrm{Se} @ \mathrm{SiO}_{2}$ nanocomposites on osteonecrosis. The results showed that compared with normal bones, MPS induced osteonecrosis (Figure 4). Importantly, although $\mathrm{Se} @ \mathrm{SiO}_{2}$ nanocomposites did not completely cure osteonecrosis, the necrotic area in the osteonecrotic bones from MPS-treated rats was significantly reduced by $\mathrm{Se} @ \mathrm{SiO}_{2}$ nanocomposite therapy (Figure 4).

\section{$\mathrm{Se} @ \mathrm{SiO}_{2}$ nanocomposites protect the cartilage of femoral head from MPS- induced injury}

Western blot was also used to determine the levels of IL-1 $\beta$, collagen II, and aggrecan in the control and $\mathrm{Se} @ \mathrm{SiO}_{2}$ groups. IL-1 $\beta$ served as the damage index in the cartilage that objectively evaluates the necrosis situation (Figure 5). Collagen II and aggrecan were properly constructed and functional proteins in the cartilage (Figure 5). Changes in the levels of IL-1 $\beta$, collagen II, and aggrecan reflected the injuries to the cartilage of the femoral head (Figure 5). The results showed that compared with the control MPS group, $\mathrm{Se} @ \mathrm{SiO}_{2}$ significantly reduced the IL- $1 \beta$ level induced by MPS (Figure 5). $\mathrm{Se} @ \mathrm{SiO}_{2}$ increased collagen II and aggrecan dramatically in the MPS-treated group (Figure 5).

\section{In vivo assessment of safety of $\mathrm{Se} @ \mathrm{SiO}_{2}$ nanocomposites}

H\&E staining and collagen II staining were used for the structural observation of the femur head. MPS induced serious destruction of the femur head. As shown in Figure 6, compared with the control MPS-induced group, the porous $\mathrm{Se} @ \mathrm{SiO}_{2}$ nanocomposite could obviously alleviate MPSinduced destruction in the bone structure (Figure 6). A low-attenuation section in the femoral head, referring to the collapse and necrosis situation it was an observed decrease in the $\mathrm{Se} @ \mathrm{SiO}_{2}$ plus MPS group compare to the MPS group. 

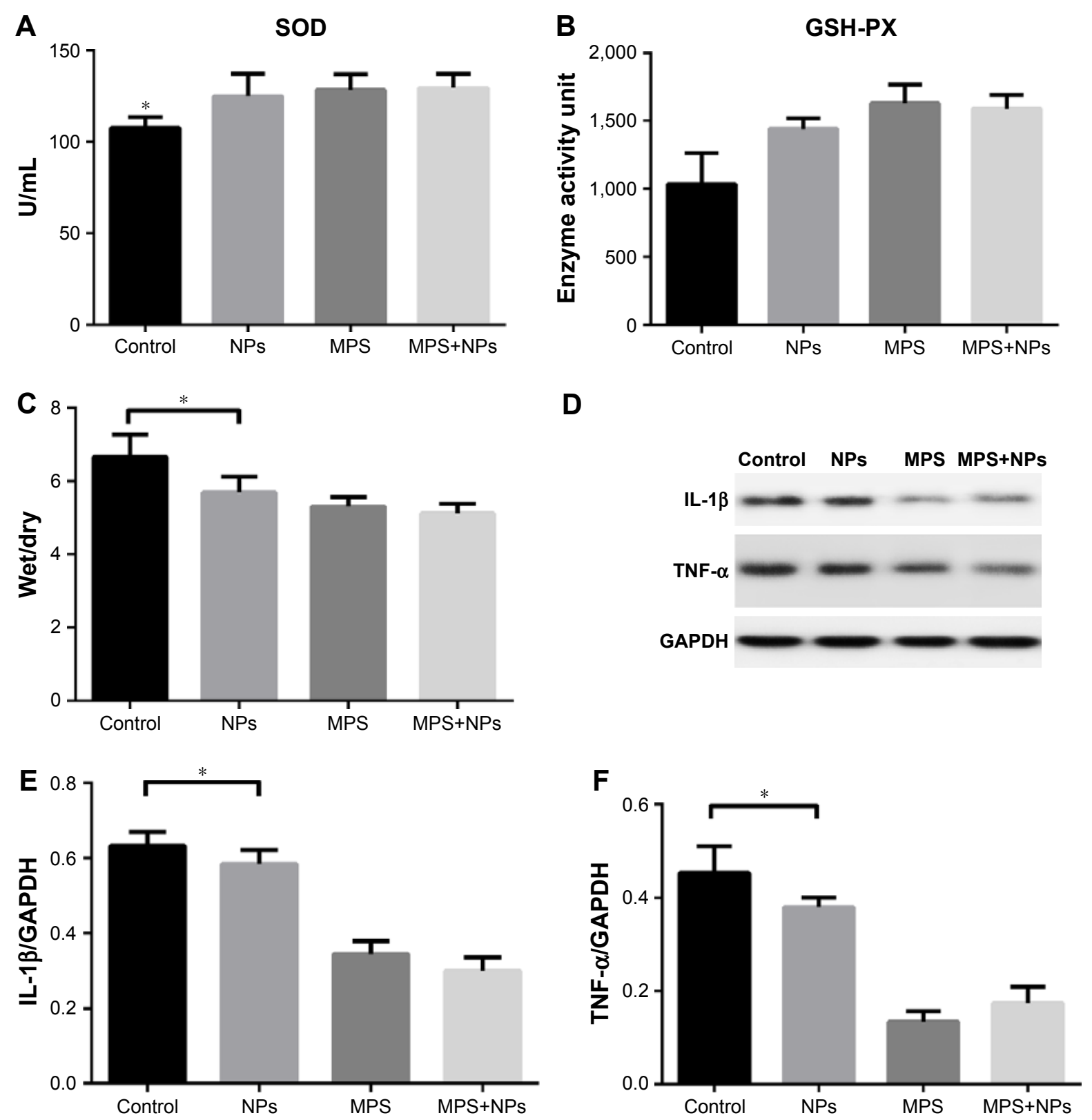

Figure 2 Porous Se@SiO nanocomposites did not impair the therapeutic efficacy of MPS in a rat model of ARDS.

Notes: (A, B) The levels of SOD and GSH in the rat lung tissues were detected with a SOD assay kit and a reduced GSH assay kit, respectively. (C) The wet-to-dry weight ratio was calculated by dividing the wet weight by the dry weight. (D) Western blot analysis of IL-I $\beta$, TNF- $\alpha$, and GAPDH expression of subchondral bone in the indicated groups. (E, F) The relative densities of (E) IL-I $\beta$ and (F) TNF- $\alpha$ proteins were normalized to GAPDH. All experiments were performed in triplicate ( $n=3$ ). Error bars represent mean \pm SEM. $* P<0.05$.

Abbreviations: ARDS, acute respiratory distress syndrome; GSH, glutathione; IL, interleukin; MPS, methylprednisolone; SEM, standard error of mean; SOD, superoxide dismutase; TNF- $\alpha$, tumor necrosis factor-alpha; NPs, nanoparticles.

The histologic analysis of tissues from the heart, liver, spleen, lung, and kidney showed that the structures in those tissues in the $\mathrm{Se} @ \mathrm{SiO}_{2}+\mathrm{MPS}$ group were comparable to those in the MPS group, suggesting the in vivo safety of $\mathrm{Se} @ \mathrm{SiO}_{2}$ (Figure 6).

\section{Discussion}

MPS is widely used as an essential drug in the clinical treatment of many diseases including ARDS. Despite the great success, MPS damages the femoral head seriously, which is irreversible. Thus, avoiding the occurrence of ONFH is more important than exploring its effective treatments.

Our study showed that $\mathrm{Se} @ \mathrm{SiO}_{2}$ nanocomposites released a beneficial amount of Se slowly. The controlled release and the in vivo stability of $\mathrm{Se} @ \mathrm{SiO}_{2}$ nanocomposites contribute to biosafety and low toxicity. ${ }^{18,21-23}$ In addition, we also examined the main organs and found that the administration 


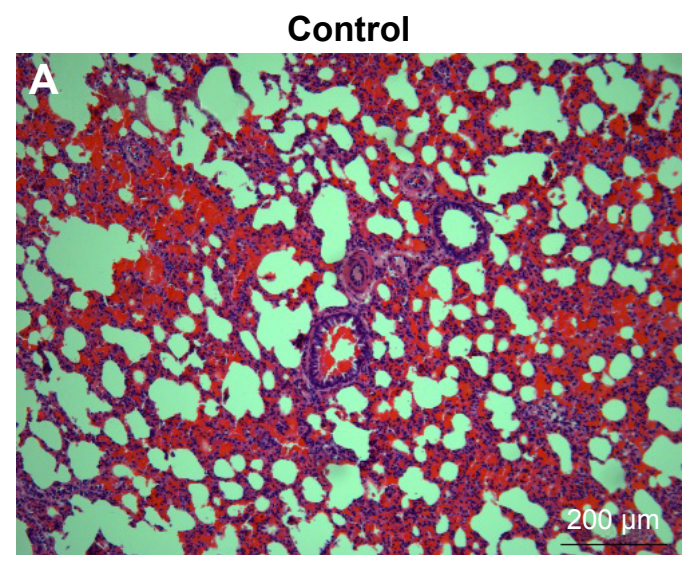

MPS

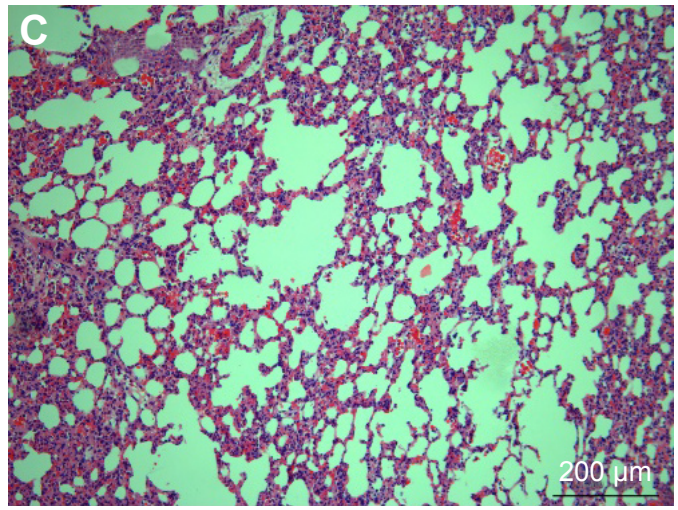

NPs

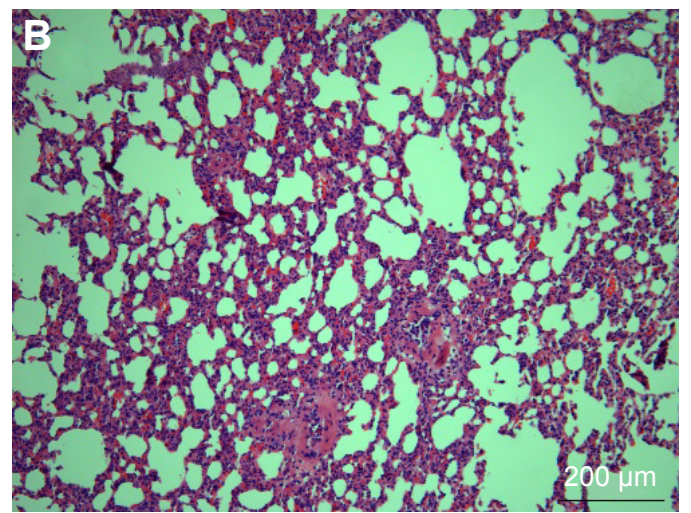

MPS+NPs

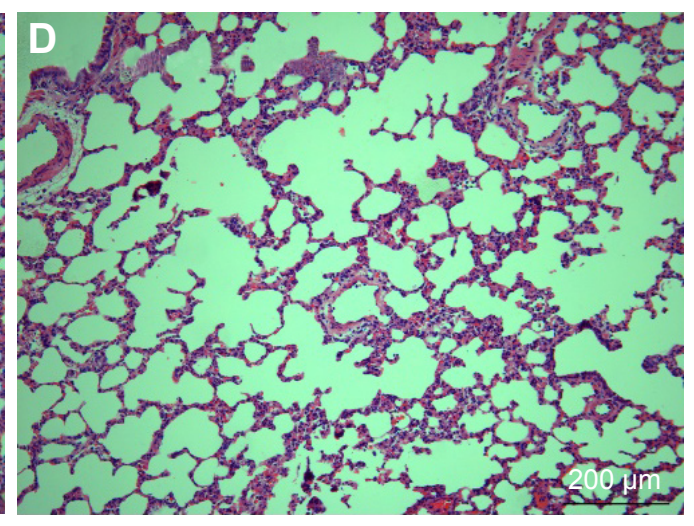

200x

Figure $3 \mathrm{Se@SiO}{ }_{2}$ nanocomposites did not reduce the injury of MPS on lung tissue. Compare to the control (A), both NPs (B) and MPS (C) can decrease the inflammatory response in lung. The combine use of NPs and MPS (D) have no significant difference to MPS group (C).

Notes: Histologic analysis of rat lung tissues. The rat lung tissues were stained with H\&E.

Abbreviations: MPS, methylprednisolone; NPs, nanoparticles.
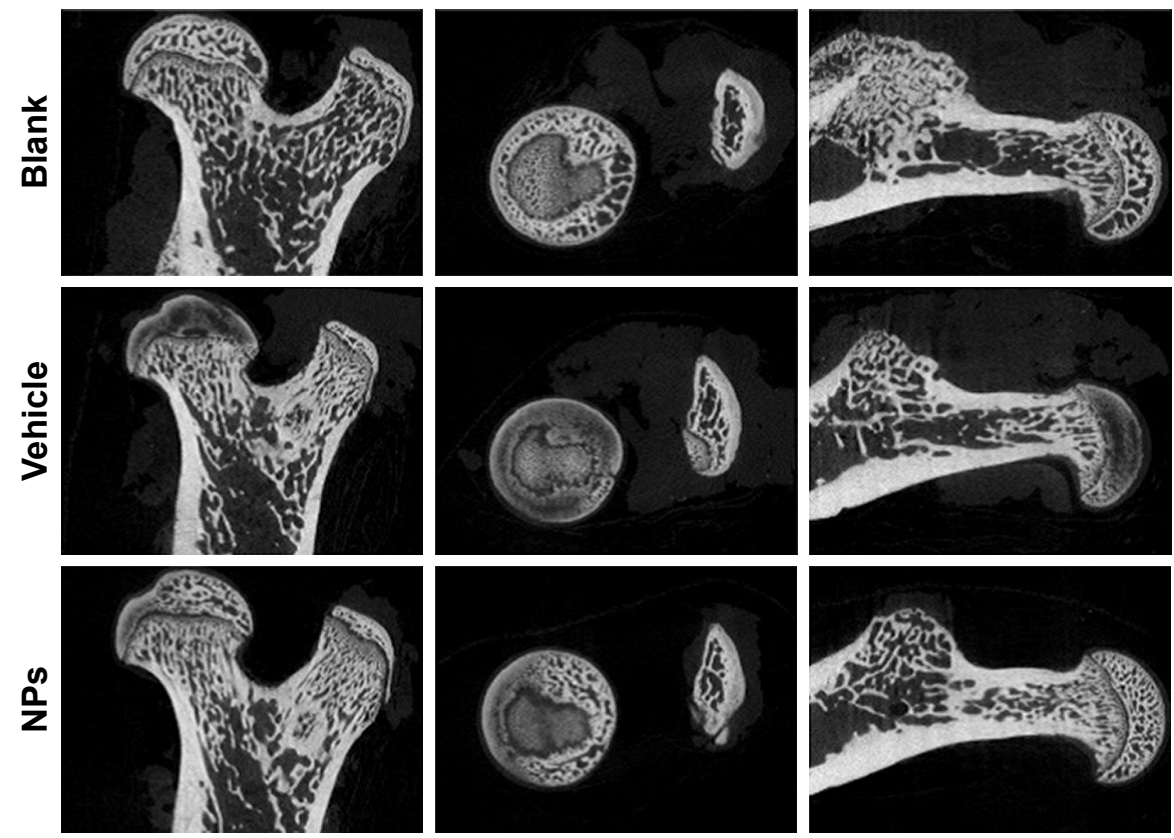

Figure $4 \mathrm{Se@SiO}$, nanocomposites reduced MPS-induced osteonecrosis in the femoral head

Note: Two-dimensional pictures of normal bone from normal rats and osteonecrotic bones from rats in the MPS and MPS+Se@SiO ${ }_{2}$ groups. Abbreviations: MPS, methylprednisolone; NPs, nanoparticles. 
A
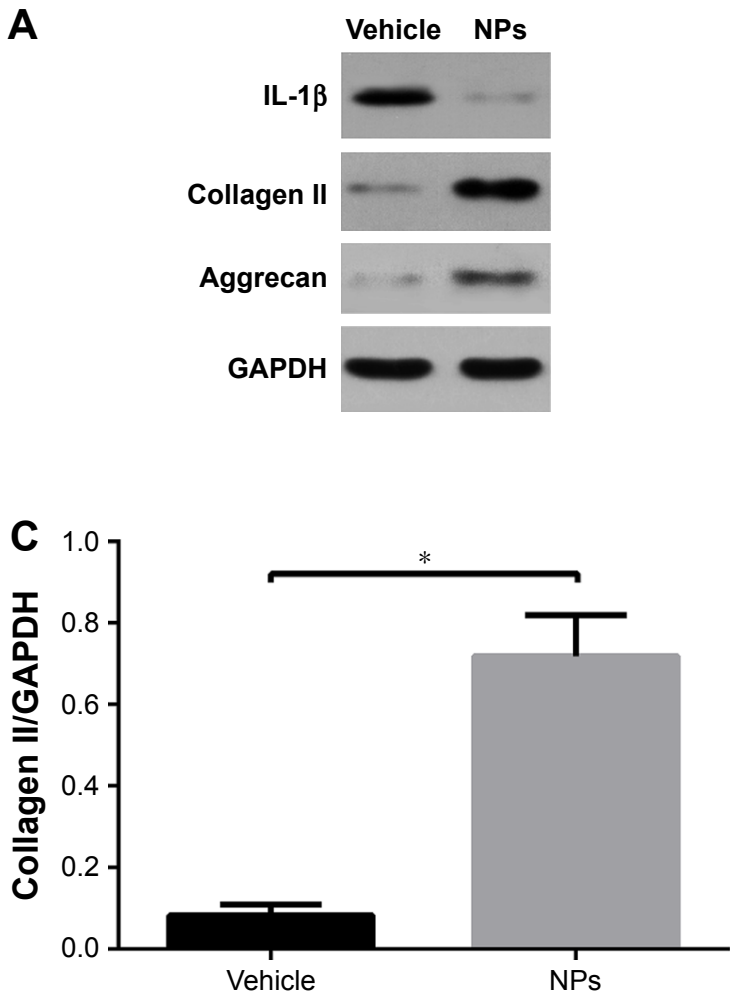
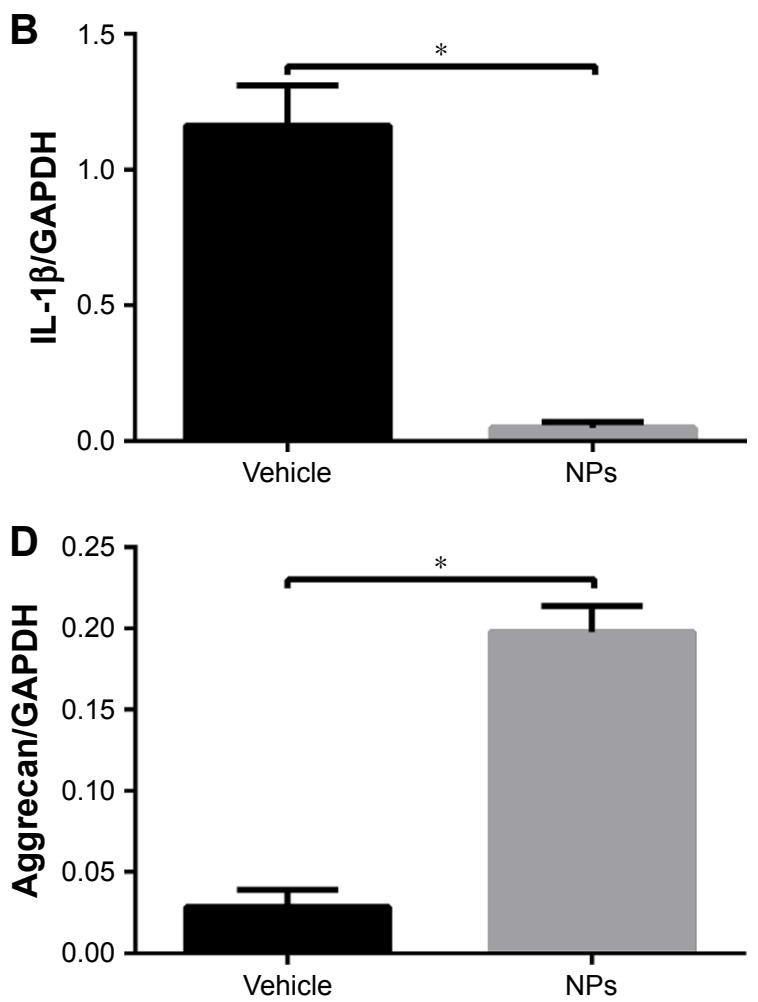

Figure $5 \mathrm{Se@SiO}$, nanocomposites protect the cartilage of femoral head from MPS-induced injury. Western blot analysis of IL-I $\beta$, collagen type II, aggrecan, and GAPDH expression of subchondral bone in the indicated groups (A). These were normalized by GAPDH and compared in B (IL-I $\alpha$ expression), $\mathbf{C}$ (collagen type II expression) and D (aggrecan expression). All three index got well with $\mathrm{Se@SiO}$, nanocomposites application, $* \mathrm{P}<0.05$.

Note: Western blot analysis of IL-I $\beta$, collagen type II, aggrecan, and GAPDH expression of subchondral bone in the indicated groups.

Abbreviations: IL, interleukin; MPS, methylprednisolone; NPs, nanoparticles.

of $\mathrm{Se} @ \mathrm{SiO}_{2}$ did not lead to any noticeable damage in these organs. Thus, the in vitro and in vivo biosafety of $\mathrm{Se} @ \mathrm{SiO}_{2}$ nanocomposites may offer them ideal properties for further use in clinical treatments.

In diseases that need MPS treatments, such as ARDS, oxidative stress is thought of as the initiating and the main mechanism. ${ }^{24}$ SOD and GSH are the principal endogenous antioxidants, which effectively remove the superoxide anion, reduce the oxidative response, and inhibit subsequent acute inflammatory response. ${ }^{25,26}$ Our study showed that $\mathrm{Se} @ \mathrm{SiO}_{2}$ has the same therapeutic effects in LPS-induced ARDS model as in paraquat-induced ARDS. Se $@ \mathrm{SiO}_{2}$ nanocomposites induced the production of SOD and GSH-PX, compared with the control group. The levels of SOD and GSH-PX in the MPS+Se@SiO 2 group were similar to those in the MPS group. In addition, the proinflammatory cytokines TNF- $\alpha$ and IL-1 $\beta$ in injured lung tissues were reduced by MPS. The addition of $\mathrm{Se} @ \mathrm{SiO}_{2}$ did not affect the inhibitory effect of MPS on both TNF- $\alpha$ and IL-1 $\beta$ levels. The wet-to-dry weight ratio of the lung tissues was reduced after porous $\mathrm{Se} @ \mathrm{SiO}_{2}$ or MPS treatment. $\mathrm{Se} @ \mathrm{SiO}_{2}$ did not impair the beneficial effect of MPS. The histologic images of rat lung tissues showed that
$\mathrm{Se} @ \mathrm{SiO}_{2}$ or MPS treatment alleviated the lung tissue damage caused by LPS, whereas the combination of $\mathrm{Se} @ \mathrm{SiO}_{2}$ and MPS further protected the lung tissues from damage. Thus, $\mathrm{Se} @ \mathrm{SiO}_{2}$ did not impair the therapeutic effect of MPS in the rat models of ARDS. In some aspects, $\mathrm{Se} @ \mathrm{SiO}_{2}$ and MPS may achieve a synergistically protective effect.

Previous studies have shown that chronic use of MPS may increase damage by ROS..$^{27}$ The induction of ROS may be the fundamental cause of ONFH. Antioxidants have a preventive effect in MPS-induced osteonecrosis in rat models. In our study, we found that $\mathrm{Se} @ \mathrm{SiO}_{2}$ nanocomposites promoted the production of the main endogenous antioxidants, including SOD and GSH. Thus, $\mathrm{Se} @ \mathrm{SiO}_{2}$ nanocomposites reduced the ROS level indirectly. Our previous studies also suggested that $\mathrm{Se} @ \mathrm{SiO}_{2}$ nanocomposites' own antioxidant property could interfere with the disease process of ONFH. ${ }^{19}$ Therefore, $\mathrm{Se} @ \mathrm{SiO}_{2}$ may have a preventive effect to be used in protection of the femoral head in MPS treatment. Here, we found that $\mathrm{Se} @ \mathrm{SiO}_{2}$ nanocomposites, indeed, alleviated MPS-induced destruction in the bone structure. IL- $1 \beta$ is a classical damage index in the cartilage that can be used objectively to evaluate the degree of necrosis. The increase 

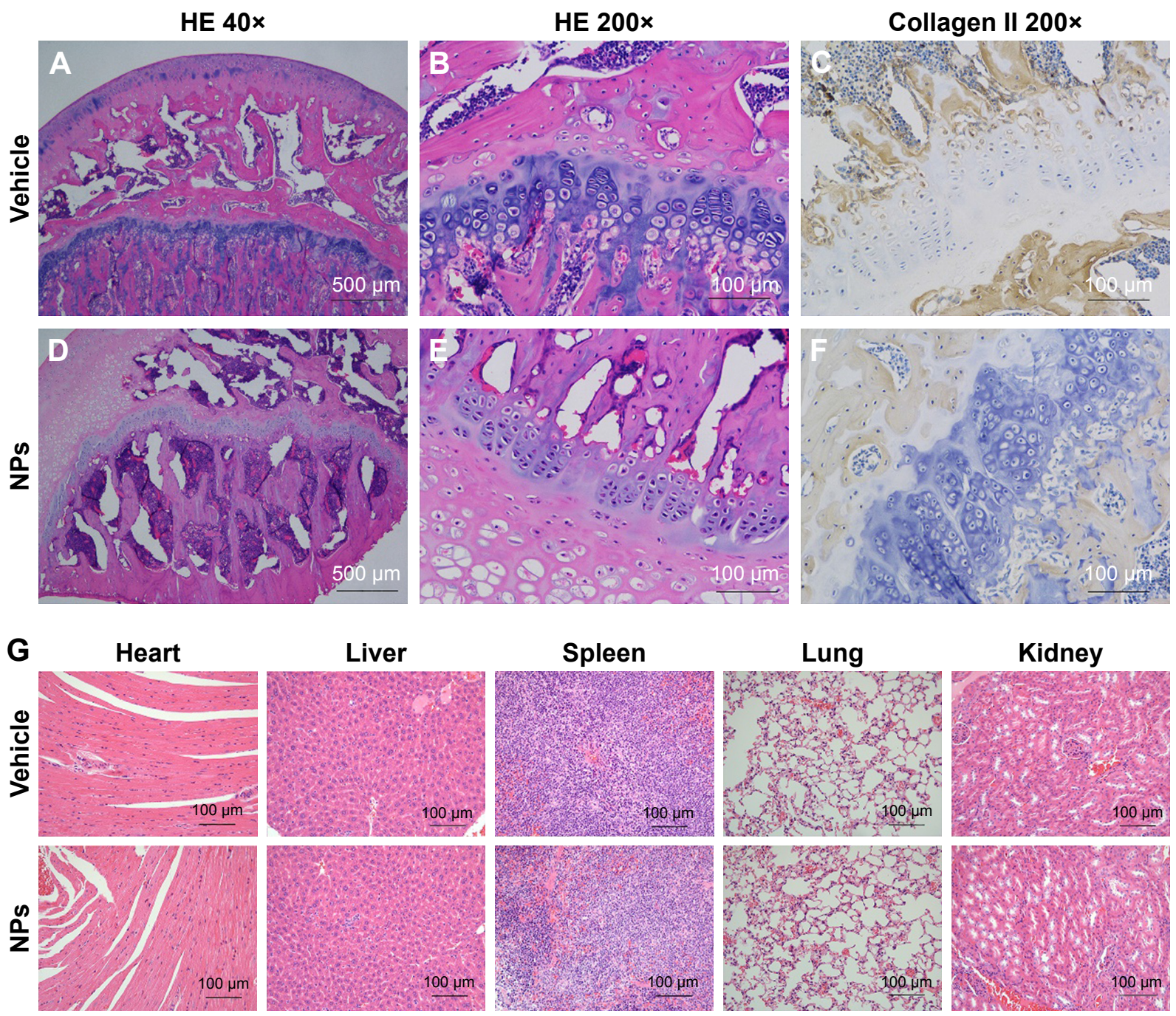

Figure 6 The in vivo assessment of safety of $\mathrm{Se} @ \mathrm{SiO}_{2}$ nanocomposites.

Notes: (A-F) Histologic images demonstrating MPS-induced femoral head necrosis with or without Se@SiO ${ }_{2}$ nanocomposite treatment. (G) Histologic images of various organs from rats treated with MPS or MPS+Se@SiO .

Abbreviations: MPS, methylprednisolone; NPs, nanoparticles.

of IL-1 $\beta$ promotes inflammation. Our study showed that compared with the control MPS group, $\mathrm{Se} @ \mathrm{SiO}_{2}$ significantly reduced the IL-1 $\beta$ level induced by MPS in the cartilage. While collagen type II and aggrecan are both properly constructed and functional proteins in the cartilage, the levels of aggrecan and collagen type II were reduced in the femoral head after MPS treatment. However, $\mathrm{Se} @ \mathrm{SiO}_{2}$ increased collagen II and aggrecan dramatically after MPS treatment. Moreover, the protective effect of $\mathrm{Se} @ \mathrm{SiO}_{2}$ in MPS-induced ONFH was further confirmed intuitively by a Micro-CT scan and histologic test.

\section{Conclusion}

Our study showed that $\mathrm{Se} @ \mathrm{SiO}_{2}$ nanocomposites exhibited a protective effect in MPS-induced ONFH without impairing the therapeutic activity of MPS. These results suggest that $\mathrm{Se} @ \mathrm{SiO}_{2}$ nanocomposites have potential as effective drugs to minimize ONFH in MPS therapy.

\section{Acknowledgments}

This study was funded by the Post-Graduation Innovation Subject of Shanghai Jiao Tong University (BXJ201734), Fund for Construction of Trauma Center of Shanghai First People's Hospital (No. 1304), and Songjiang District Trauma Linkage System Construction fund (0702N14004).

\section{Author contributions}

All authors contributed toward data analysis, drafting and critically revising the paper and agree to be accountable for all aspects of the work. 


\section{Disclosure}

The authors report no conflicts of interest in this work.

\section{References}

1. El Gamal TA, El-Bakoury A, Hawkins A, Ed AlTayeb Mussa M, Er Ahmed Sweed T, Eh Samir Ansara S. Bilateral osteonecrosis of the femoral and humeral heads after short term corticosteroid therapy. A Case Study. Ortop Traumatol Rehabil. 2016;18(2):187-190.

2. Issa K, Pivec R, Kapadia BH, Banerjee S, Mont MA. Osteonecrosis of the femoral head: the total hip replacement solution. Bone Joint J. 2013;95-B(11 Suppl A):46-50.

3. Puppel K, Kapusta A, Kuczynska B. The etiology of oxidative stress in the various species of animals, a review. J Sci Food Agric. 2015;95(11): 2179-2184.

4. Ichiseki T, Kaneuji A, Ueda Y, et al. Osteonecrosis development in a novel rat model characterized by a single application of oxidative stress. Arthritis Rheum. 2011;63(7):2138-2141.

5. Komurcu E, Oktay M, Kaymaz B, Hatay Golge U, Goksel F, Nusran G. Preventive effects of coenzyme Q10 (CoQ10) on steroid-induced osteonecrosis in rats. Acta Orthop Traumatol Turc. 2014;48(2):217-222.

6. Huang SL, Jiao J, Yan HW. Hydrogen-rich saline attenuates steroidassociated femoral head necrosis through inhibition of oxidative stress in a rabbit model. Exp Ther Med. 2016;11(1):177-182.

7. Gil HW, Hong JR, Jang SH, Hong SY. Diagnostic and therapeutic approach for acute paraquat intoxication. J Korean Med Sci. 2014; 29(11):1441-1449.

8. Kahya MC, Naziroglu M, Cig B. Melatonin and selenium reduce plasma cytokine and brain oxidative stress levels in diabetic rats. Brain Inj. 2015;29(12):1490-1496.

9. Ebert R, Ulmer M, Zeck S, et al. Selenium supplementation restores the antioxidative capacity and prevents cell damage in bone marrow stromal cells in vitro. Stem Cells. 2006;24(5):1226-1235.

10. Kim JH, Kang JC. Oxidative stress, neurotoxicity, and non-specific immune responses in juvenile red sea bream, Pagrus major, exposed to different waterborne selenium concentrations. Chemosphere. 2015; 135:46-52.

11. Stohs SJ, Bagchi D. Oxidative mechanisms in the toxicity of metal ions. Free Radic Biol Med. 1995;18(2):321-336.

12. McDonald CI, Fung YL, Shekar K, et al. The impact of acute lung injury, ECMO and transfusion on oxidative stress and plasma selenium levels in an ovine model. J Trace Elem Med Biol. 2015;30:4-10.

13. Yao L, Du Q, Yao H, Chen X, Zhang Z, Xu S. Roles of oxidative stress and endoplasmic reticulum stress in selenium deficiency-induced apoptosis in chicken liver. Biometals. 2015;28(2):255-265.
14. Wang Y, Chen P, Zhao G, et al. Inverse relationship between elemental selenium nanoparticle size and inhibition of cancer cell growth in vitro and in vivo. Food Chem Toxicol. 2015;85:71-77.

15. Sun HJ, Rathinasabapathi B, Wu B, Luo J, Pu LP, Ma LQ. Arsenic and selenium toxicity and their interactive effects in humans. Environ Int. 2014;69:148-158.

16. Zalavras CG, Lieberman JR. Osteonecrosis of the femoral head: evaluation and treatment. J Am Acad Orthop Surg. 2014;22(7):455-464.

17. Hassanin KM, Abd El-Kawi SH, Hashem KS. The prospective protective effect of selenium nanoparticles against chromium-induced oxidative and cellular damage in rat thyroid. Int J Nanomedicine. 2013;8: $1713-1720$.

18. Liu X, Deng G, Wang Y, et al. A novel and facile synthesis of porous $\mathrm{SiO} 2$-coated ultrasmall Se particles as a drug delivery nanoplatform for efficient synergistic treatment of cancer cells. Nanoscale. 2016; $8(16): 8536-8541$.

19. Deng G, Niu K, Zhou F, et al. Treatment of steroid-induced osteonecrosis of the femoral head using porous $\mathrm{Se} @ \mathrm{SiO} 2$ nanocomposites to suppress reactive oxygen species. Sci Rep. 2017;7:43914.

20. Jiang Y, Zhang Y, Chen W, et al. Achyranthes bidentata extract exerts osteoprotective effects on steroid-induced osteonecrosis of the femoral head in rats by regulating RANKL/RANK/OPG signaling. J Transl Med. 2014; $12: 334$

21. Zhang J, Wang X, Xu T. Elemental selenium at nano size (Nano-Se) as a potential chemopreventive agent with reduced risk of selenium toxicity: comparison with se-methylselenocysteine in mice. Toxicol Sci. 2008;101(1):22-31.

22. Wang $\mathrm{H}$, Zhang J, Yu H. Elemental selenium at nano size possesses lower toxicity without compromising the fundamental effect on selenoenzymes: comparison with selenomethionine in mice. Free Radic Biol Med. 2007;42(10):1524-1533.

23. He Y, Chen S, Liu Z, Cheng C, Li H, Wang M. Toxicity of selenium nanoparticles in male Sprague-Dawley rats at supranutritional and nonlethal levels. Life Sci. 2014;115(1-2):44-51.

24. Zhu Y, Deng G, Ji A, et al. Porous Se@SiO2 nanospheres treated paraquat-induced acute lung injury by resisting oxidative stress. Int $J$ Nanomedicine. 2017;12:7143-7152.

25. Yamashita M, Yamashita M, Ando Y. A long-term follow-up of lung function in survivors of paraquat poisoning. Hum Exp Toxicol. 2000;19(2):99-103.

26. Yoon SC. Clinical outcome of paraquat poisoning. Korean J Intern Med. 2009;24(2):93-94.

27. Torres RL, Torres IL, Laste G, Ferreira MB, Cardoso PF, Bello-Klein A. Effects of acute and chronic administration of methylprednisolone on oxidative stress in rat lungs. J Bras Pneumol. 2014;40(3):238-243.
International Journal of Nanomedicine

\section{Publish your work in this journal}

The International Journal of Nanomedicine is an international, peerreviewed journal focusing on the application of nanotechnology in diagnostics, therapeutics, and drug delivery systems throughout the biomedical field. This journal is indexed on PubMed Central, MedLine, CAS, SciSearch ${ }^{\circledR}$, Current Contents ${ }^{\circledR} /$ Clinical Medicine,

\section{Dovepress}

Journal Citation Reports/Science Edition, EMBase, Scopus and the Elsevier Bibliographic databases. The manuscript management system is completely online and includes a very quick and fair peer-review system, which is all easy to use. Visit http://www.dovepress.com/ testimonials.php to read real quotes from published authors. 